\title{
Lossless image compression via predictive coding of discrete Radon projections
}

\author{
Andrew Kingston ${ }^{\mathrm{a}, \mathrm{b}}$, Florent Autrusseau ${ }^{\mathrm{a}, *}$ \\ a IRCCyN lab. Polytech'Nantes, Rue Ch. Pauc, BP 50609, 44306 Nantes, France \\ ${ }^{\mathrm{b}}$ Department of Applied Mathematics, Research School of Physical Sciences and Engineering, Australian National University, Canberra, ACT 0200, Australia
}

\section{A R T I C L E I N F O}

\section{Article history:}

Received 20 November 2007

Accepted 11 March 2008

\section{Keywords:}

Lossless image coding

Discrete Radon transform

Mojette

Redundancy

\begin{abstract}
A B S T R A C T
This paper investigates predictive coding methods to compress images represented in the Radon domain as a set of projections. Both the correlation within and between discrete Radon projections at similar angles can be exploited to achieve lossless compression. The discrete Radon projections investigated here are those used to define the Mojette transform first presented by Guédon et al. [Psychovisual image coding via an exact discrete Radon transform, in: T.W. Lance (Ed.), Proceedings of the Visual Communications AND Image Processing (VCIP), May 1995, Taipei, Taiwan, pp. 562-572]. This work is further to the preliminary investigation presented by Autrusseau et al. [Lossless compression based on a discrete and exact radon transform: a preliminary study, in: Proceedings of the IEEE International Conference on Acoustics, Speech, and Signal Processing (ICASSP), vol. II, May 2006, Toulouse, France, pp. 425-428]. The 1D Mojette projections are re-arranged as two dimensional images, thus allowing the use of 2D image compression techniques onto the projections. Besides the compression capabilities, the Mojette transforms brings an interesting property: a tunable redundancy. As the Mojette transform is able to both compress and add redundancy, the proposed method can be viewed as a joint lossless source-channel coding technique for images. We present here the evolution of the compression ratio depending on the chosen redundancy.
\end{abstract}

(c) 2008 Elsevier B.V. All rights reserved.

\section{Introduction}

This work is motivated by the limitations of image processing tools in large multi-media databases. Numerous paintings belonging to French museums are stored in an image database within the 'Centre of Research and Restoration of French Museums' (C2RMF). ${ }^{1}$ It is required that these high payload (up to 1000 Megapixel) images be losslessly compressed, stored securely (i.e., with some redundancy) and encrypted for transmission purposes. The French TSAR project (Secure Transfer of High Resolution Art Images ${ }^{2}$ ) aims to develop a method to securely

\footnotetext{
* Corresponding author.

E-mail address: Florent.Autrusseau@univ-nantes.fr (F. Autrusseau).

${ }^{1}$ http://www.c2rmf.fr

2 http://www.lirmm.fr/tsar/
}

transfer images from the image database of artwork contained in the Louvre Museum. All this can also be said of medical image databases. It has been shown that the Mojette transform can be used for distributed storage [7] and encryption [10], if competitive lossless compression can also be achieved on the Mojette projection data, the majority of the above objectives can be achieved using only the Mojette transform. Although joint sourcechannel coding has been extensively studied in the literature [20], most of the interest has been focused on lossy coding [3] with only limited research being conducted on lossless joint source-channel coding for images [2]. Applications in geophysics and telemetry as well as the previously mentioned museum and medical image databases all require lossless compression [13]. We do not focus here on the properties of the Mojette transform compared to state of the art joint sourcechannel coding, but our goal is rather to improve 
a previous study [1] in terms of lossless compression rate. We nevertheless want to point out the important link between the Mojette transform and maximum distance separable (MDS) codes used for joint source-channel coding (e.g. Reed-Solomon or BCH codes). The performances of the proposed algorithm regarding both compression and redundancy will be shown in Section 4.1.

The Mojette transform is an entirely discrete mapping (from a discrete image to discrete projections), which requires only the addition operation and is exactly invertible. It retains the major properties of the Radon transform such as the Fourier slice theorem and the related convolution property but also introduces new properties such as redundancy. It was first proposed by Guédon et al. in 1995 [5] in the context of psychovisual image coding. It has since been applied in many aspects of image processing such as image analysis, image watermarking, image encryption, and tomographic image reconstruction from projections. The unique properties of the transform have also made it a useful multiple description tool with applications in robust data transmission and distributed data storage. A summary of the evolution and applications of the Mojette transform to date can be found in [6].

Since the Mojette transform already has other advantages, the objective of this work is not to develop a superior image compression standard. Rather, we seek to extend the work by Autrusseau et al. in [1] on the compression of Mojette projection data to become comparable with results from existing techniques. The preliminary study introduced the idea of a compression scheme which exploits correlation within a projection (intra-projection coding) as well as compression scheme which exploits correlation between projections (interprojection coding). Since the Mojette projections are principally used in a data transmission and storage context, developing compression techniques which are effective is important. Both types of coding must be investigated as, if redundant projections are required in the transform, only intra-projection coding may be possible. This paper investigates several methods to compress projections by adapting multi-spectrum image compression techniques to multi-projection data.

The paper is organised as follows: The Mojette transform, projection properties and inverse are presented in the next section. A summary of the preliminary study by Autrusseau et al. [1] follows in Section 3. Section 4 demonstrates how inter-band image compression techniques can be applied. This is followed by some concluding remarks and future research directions in Section 5.

\section{A discrete Radon transform: The Mojette transform}

\subsection{Mojette projections}

The Radon transform ${ }^{3}$ maps a continuous 2D function to a set of $1 \mathrm{D}$ continuous projections at all angles $\theta \in[0, \pi)$.

${ }^{3}$ Ref. [4] provides an English translation of Radon's original 1917 paper.
A projection at angle, $\theta$, is obtained as the linear integration of the function over all parallel lines with gradient $\tan \theta$. One of the most important properties of the Radon transform is that it is invertible. This implies that the internal structure of an object can be determined nondestructively from its projections (tomography). The Radon transform is utilised in areas ranging from medical tomography (CT, MRI, ultrasound) to astronomy and seismology. In recent years it has also been applied to many aspects of image analysis, image representation and image processing. Since the projection data and reconstructed image are both discrete, the implementation of the Radon transform and its inverse must be discretised. Many methods involve filtering and interpolating the discrete data; a numerically intensive procedure. There have also been several discrete Radon transforms proposed which naturally deal with discrete data, e.g. [11,9]. This paper is concerned with one particular discrete Radon transform known as the Mojette transform.

The Mojette transform is an exact, discrete form of the Radon transform defined for specific 'rational' projection angles. Like the classical Radon transform, the Mojette transform represents the image as a set of projections, however, in contrast, the Mojette transform has an exact inverse from a finite number of discrete projections (as few as 1 depending on the angle set). The rational projection angles, $\theta_{i}$, are defined by a set of vectors $\left(p_{i}, q_{i}\right)$ as $\theta_{i}=\tan ^{-1}\left(q_{i} / p_{i}\right)$, as depicted in Fig. 1 a for $\left(p_{i}, q_{i}\right)=(2,1)$. These vectors must respect the condition that $p_{i}$ and $q_{i}$ are coprime (i.e., $\operatorname{gcd}\left(p_{i}, q_{i}\right)=1$ ) and since $\tan$ is $\pi$-periodic $q_{i}$ is restricted to be positive except for the case $\left(p_{i}, q_{i}\right)=(1,0)$. The transform domain of an image is a set of projections where each element (called a 'bin' as in tomography) corresponds to the sum of the pixels centred on the line of projection as depicted in Fig. 1a. This is a linear transform defined for each projection angle by the operator:

$$
\begin{aligned}
\mathscr{M}_{p_{i}, q_{i}}\{f(k, l)\} & =\operatorname{proj}_{p_{i}, q_{i}}(b) \\
& =\sum_{k=-\infty}^{+\infty} f(k, l) \Delta\left(b+k q_{i}-l p_{i}\right),
\end{aligned}
$$

where $(k, l)$ defines the position of an image pixel and $\Delta(b)$ is the Kronecker delta function which is 1 when $b=0$ and zero otherwise. Invertible projections can be obtained not only with addition but using any linear operation. Other practically useful operations include modulo 256 addition and bitwise XOR. The Mojette transform, $\mathscr{M}_{I} f(k, l)$, corresponds to the set of $I$ projections as $\mathscr{M}_{I} f(k, l)=\left\{\operatorname{proj}_{p_{i}, q_{i}}\right.$, $i \in[1, \ldots, I]\}$.

As depicted for the example images in Fig. 1a and b, each bin value equals the sum of the pixels crossed by the appropriate line

$b=l p_{i}-k q_{i}$.

The principle difference from the classical Radon transform is the sampling rate on each projection, which is no longer constant but depends on the chosen angle as $1 / \sqrt{p_{i}^{2}+q_{i}^{2}}$. This can be seen for the different projections in Fig. 1b which demonstrates the Mojette transform for 
a

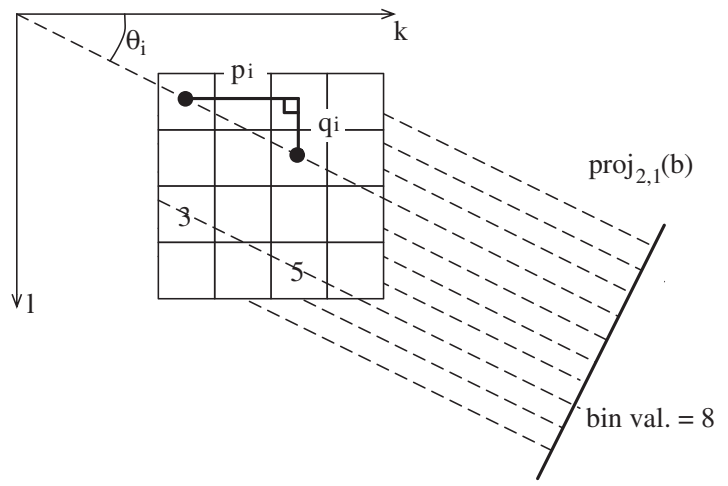

b

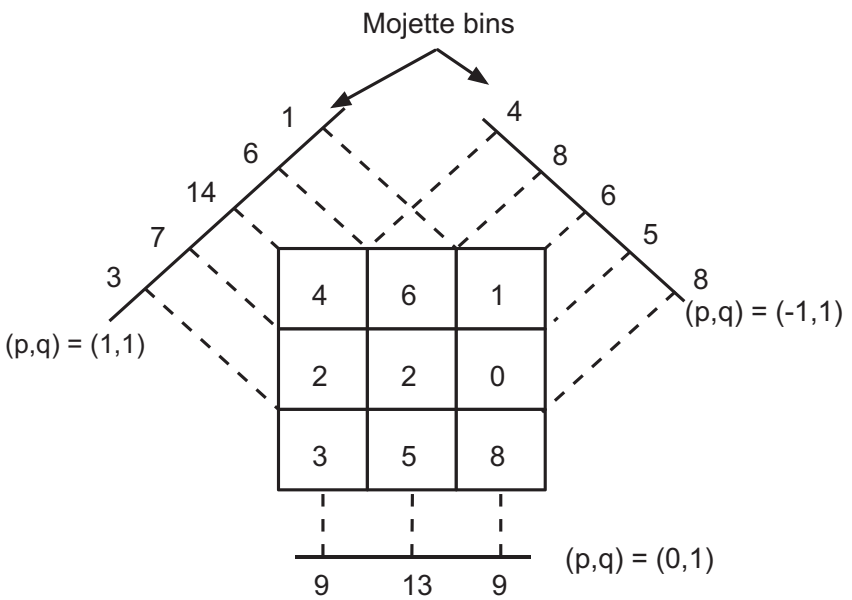

Fig. 1. (a) A depiction of $\left(p_{i}, q_{i}\right)$, the corresponding angle, $\theta_{i}$, and the method of projection, i.e., summing pixel values centred on the line to give a bin value. (b) An invertible Mojette transform of a $3 \times 3$ example image using direction vectors $\{(1,0),(1,1),(-1,1)\}$. Note the spacing between adjacent line sums varies with projection angle.
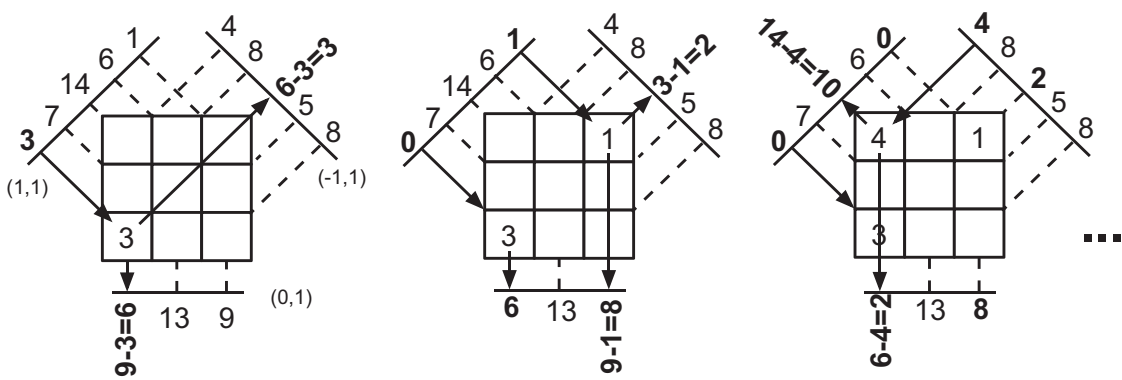

Fig. 2. Three first possible steps of the inverse Mojette transform of the projections obtained in Fig. 1.

the directions set $S=\{(1,0)(-1,1)$ and $(1,1)\}$. The number of bins, $B_{i}$, for each projection depends on the chosen direction vector $\left(p_{i}, q_{i}\right)$, and for a $P \times Q$ image is found as

$B_{i}=(Q-1)\left|p_{i}\right|+(P-1) q_{i}+1$.

The algorithmic complexity of the Mojette transform for a $P \times Q$ image with $I$ projections is $O(P Q I)$.

\subsection{Conditions for reconstructability}

Since the set of projection directions is selected arbitrarily, the original data cannot necessarily be recovered from the set of projections chosen. A criterion is required to determine if a set of projections is sufficient to uniquely reconstruct the data.

The first result on the conditions for the existence of a unique reconstruction from a given set of $I$ projections came from Katz [8] in a very similar context. He showed that if the following criterion is satisfied, any rectangular $P \times Q$ data set can be uniquely reconstructed:

$P \leqslant \sum_{i=1}^{I}\left|p_{i}\right|$ or $\quad Q \leqslant \sum_{i=1}^{I} q_{i}$.
This result has been extended in an independent manner by Normand and Guédon [14] to apply to data with compact support of any shape.

\subsection{Reconstruction from Mojette projections}

The inverse Mojette transform is a fast and simple algorithm [14]. Searching for and updating 1-1 pixel-bin correspondence enables a simple iterative procedure to recover the image. The bin value is back-projected into the pixel and subtracted from the corresponding bins in all other projections. The number of pixels belonging to the corresponding bins is also decremented. The algorithmic complexity of the inverse Mojette transform for a $P \times Q$ image with $I$ projections is $O(P Q I)$ [14]. Fig. 2 shows one possibility of the first three steps of the inverse Mojette transform of the example projections given in Fig. 1.

This 'accounting' inverse was improved by Normand et al. in [15] where the need to search for 1-1 pixel-bin correspondence was removed. It was proven that when ordered by angle, $\theta_{i}=\tan ^{-1}\left(q_{i} / p_{i}\right)$, each projection reconstructs the subsequent $q_{i}$ rows of the image for the case where $\sum q_{i}=Q$ (or subsequent $\left|p_{i}\right|$ columns where 
$\left.\sum\left|p_{i}\right|=P\right)$. This knowledge enables the periodic sequence of reconstructible pixels to be predetermined, removing the need for the accounting images in the reconstruction.

This result improves reconstruction time by a factor of 5 but is also useful for removing unwanted redundancy. Since the rows (or columns) of the image that are reconstructed by a given projection are known, any projection bins not containing pixels from these rows (or columns) can be removed. Thus for pure compression applications, the Mojette transform can be completely non-redundant mapping $P \times Q$ pixels to $P Q$ bins.

\section{A review of the preliminary study}

Autrusseau et al. [1] noted that the Mojette projection data are highly correlated within a projection and also that a strong correlation exists between projections at similar angles. This can be seen in Fig. 3a(i)-(iii) for the projection set $\{(1,171),(1,172),(1,173)\}$ with respective projection angles of $89.665^{\circ}, 89.667^{\circ}$, and $89.669^{\circ}$. This implies that a form of differential coding should be an efficient compression technique.

The technique presented in the paper defines a simple compression technique based on two differential pulse code modulation (DPCM) schemes of order 1. One scheme is applied within a projection, defined as 'intra-projection' coding, and the other is applied between projections, defined as 'inter-projection' coding.

Let $b_{n}^{i}$ denote the value of the $n$th bin of the $i$ th projection, i.e., $\operatorname{proj}_{p_{i}, q_{i}}(n)$. Assume this is the current bin value to be coded and let $\widehat{b}_{n}^{i}$ be the prediction of $b_{n}^{i}$ with the encoded prediction error defined as $e_{n}^{i}=b_{n}^{i}-\widehat{b}_{n}^{i}$.
According to (2), horizontally adjacent pixels in the image are separated by $q_{i}$ in the projection bins. Therefore the projection data are periodic with $q_{i}$ and an appropriate prediction for intra-projection coding is

$\widehat{b}_{n}^{i}=b_{n-q_{i}}^{i}$.

Fig. $3 b(i)$ depicts the result of this coding applied to the section of proj $_{1,171}$ given in Fig. 3a(ii). A prediction for inter-projection coding is simply defined as

$\widehat{b}_{n}^{i}=\widetilde{b}_{n}^{i+1}$

where $\widetilde{b}_{n}^{i+1}$ is the bin in $\operatorname{proj}_{p_{i+1}, q_{i+1}}$ that 'best' corresponds to $b_{n}^{i}$. This is more difficult to realise in practice since the projections are of different length and the most appropriate bin to utilise for prediction is not obvious. This is explored more fully in Section 4, here linear interpolation is used. Fig. $3 \mathrm{~b}$ (ii) depicts the result of this coding applied to the section of $\operatorname{proj}_{1,171}$ given in Fig. $3 a(i i)$ using proj $_{1,172}$ as the reference projection. Both schemes used together produce a DPCM of order 3 as follows:

$\widehat{b}_{n}^{i}=b_{n-q_{i}}^{i}+\widetilde{b}_{n}^{i+1}-\widetilde{b}_{n-q_{i+1}}^{i+1}$.

Fig. $3 \mathrm{~b}$ (iii) shows the result of this coding applied to the section of proj $_{1,171}$ given in Fig. $3 a$ (ii) once again using $\operatorname{proj}_{1,172}$ as the reference projection.

It is important to note that given $I$ projections, the total number of bins according to $(3)$ is $I+(Q-1) \sum\left|p_{i}\right|+$ $(P-1) \sum q_{i}$ and Katz criterion, (4), must be satisfied for inversion. This implies projection sets that minimise redundancy are those of the form $\left\{\left(1, q_{1}\right),\left(1, q_{2}\right)\right.$, $\left.\ldots,\left(1, q_{I}\right)\right\}$ such that $\sum q_{i}$ is equal to or only slightly greater than $Q$ (or $\left\{\left(p_{1}, 1\right),\left(p_{2}, 1\right), \ldots,\left(p_{I}, 1\right)\right\}$ such that a
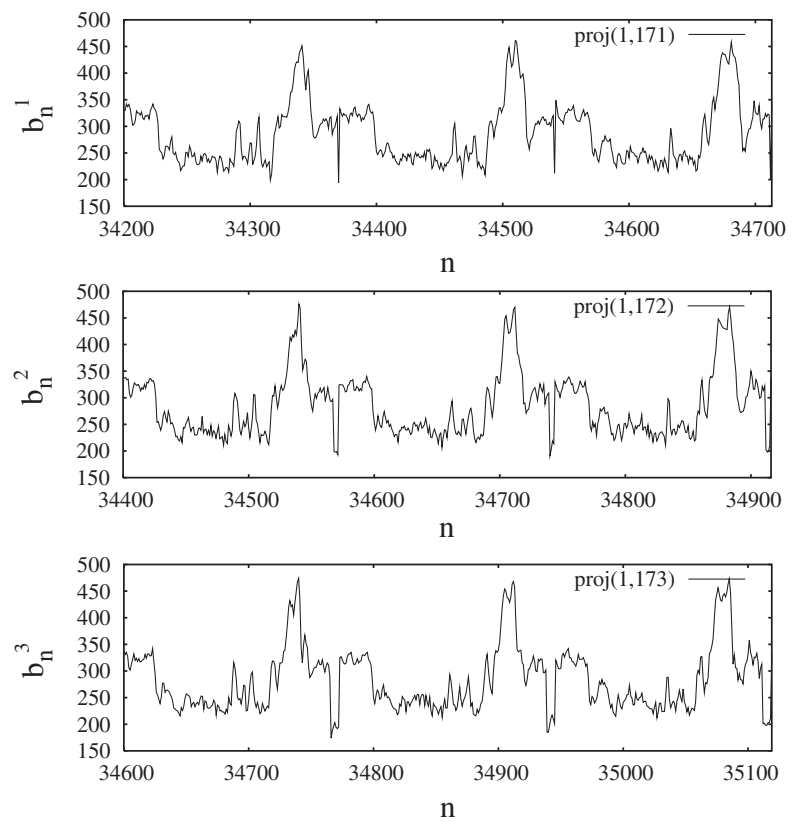

b
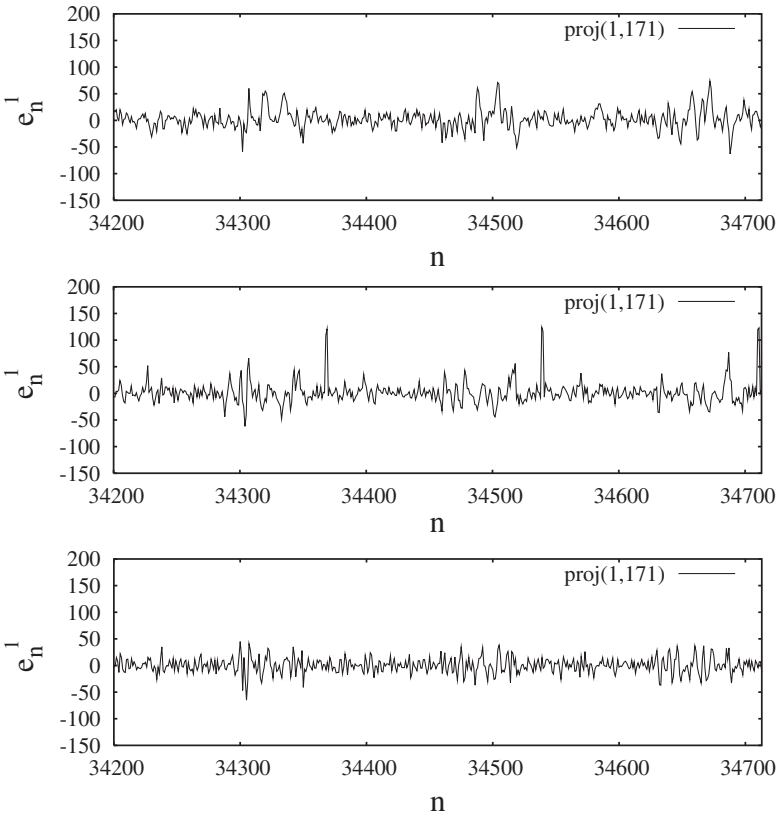

Fig. 3. (a) A zoom of three projections of 'Lena' with projection vectors $\left(1, q_{i}\right)$ where $q_{i}$ is (i) 171 , (ii) 172 , and (iii) 173 showing the periodicity within a projection and the correlation between the projections. (b) (i) Intra-projection coding applied to the section of proj ${ }_{1,171}$ given in (a(i)). (ii) Inter-projection coding using $\operatorname{proj}_{1,172}(\mathrm{a}(\mathrm{ii}))$ as a reference projection. (iii) Both intra- and inter-projection coding of proj ${ }_{1,171}$. 


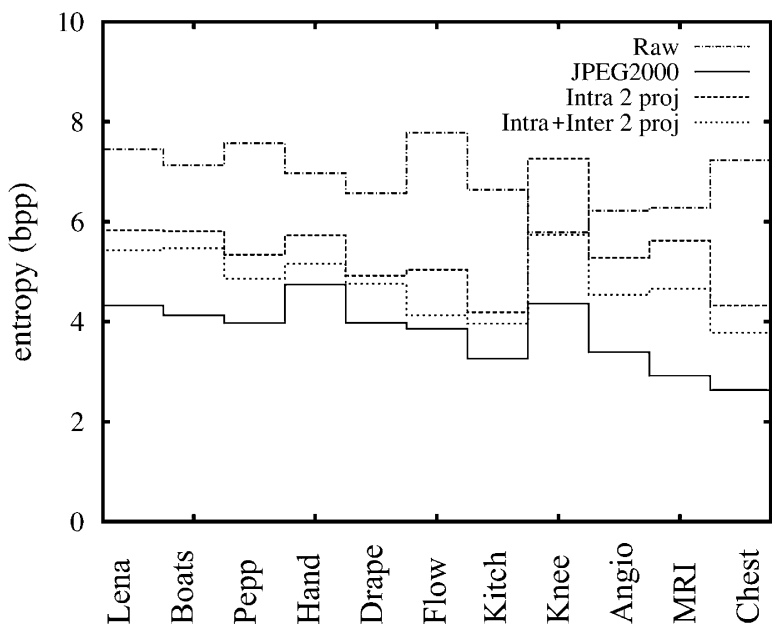

Fig. 4. (a) The compression results for intra- and inter-projection compression for two projections as outlined in [1] compared with JPEG2000 for the 11 test images depicted in (b) numbered left to right, top to bottom. The first row contains $512 \times 512$ natural images: 'Lena', 'Boats', 'Peppers'. The second row contains C2RMF images: 'Hand' $1200 \times 1854$, 'Drape' $2376 \times 3542$, 'Flowers' $1405 \times 1125$, 'Kitchen' $3822 \times 3333$. The last row contains $256 \times 256$ medical images: 'Knee', 'Angio', 'MRI', 'Chest'. $\left.\sum\left|p_{i}\right| \geqslant P\right)$. In a compression context, this restriction is more dominant than selecting projection directions according to texture orientation. A method to take advantage of this is a subject for future research.

The results of applying (5) and (7) followed by entropy coding to 11 test images has been presented in Fig. 4. Compression results have been given as the final entropy in bits per pixel (bpp) and have been compared with the original entropy of the image and the compression results from applying JPEG2000. JPEG2000 has been selected for comparison even though it does not generally give optimal compression. It is robust to image type (i.e., natural, artificial, smooth, textured), and is commonly used due to its multi-resolutional capabilities. Likewise, the Mojette also has other capabilities in data storage and encryption so achieving compression at least comparable to JPEG2000 is desirable.

Fig. 4 shows that some degree of compression is generally achieved (apart from the high contrast 'Knee' image), however, the results are not comparable to those from JPEG2000. The next section seeks to identify more powerful prediction schemes which are appropriate for both intra- and inter-projection coding to improve compression results.

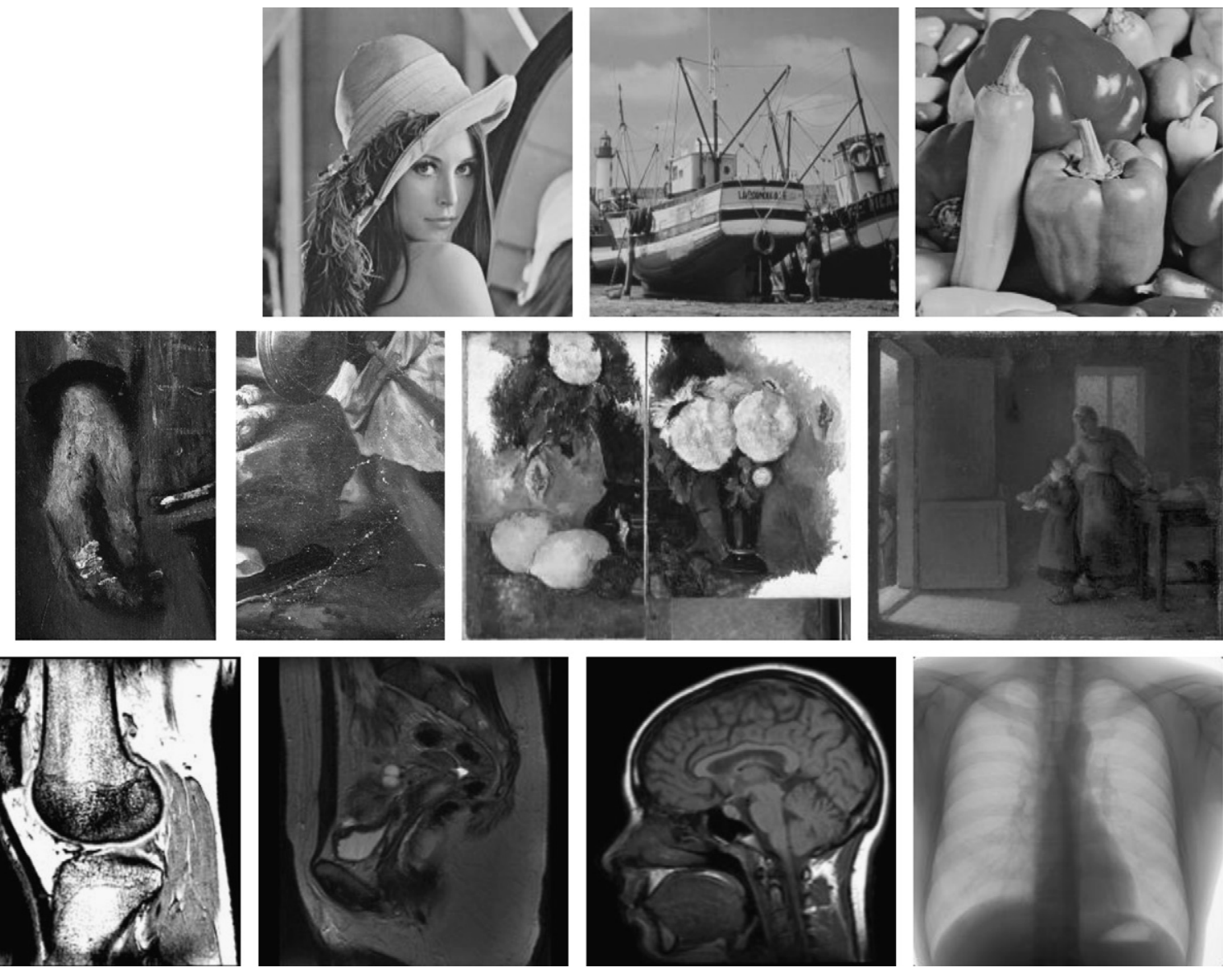

Fig. 4. (Continued) 


\section{Projection compression using multi-band image techniques}

\subsection{Intra-projection compression of projection images}

Phillipé and Guédon [16] showed that the 2D image auto-correlation is retained in the Mojette projections. If the projection data are arranged in columns of length $q_{i}$ or rows of length $p_{i}$ (whichever is greater is preferable), this auto-correlation becomes apparent as the projection appears as a 'folded' image. This has been depicted in Fig. 5 for three projections of 'Lena'. The remapping of projection bins is performed by projecting pixel value, $f(k, l)$, to $\operatorname{proj}_{p_{i}, q_{i}}\left(n_{k}, n_{l}\right)$ according to

if $\left|p_{i}\right| \geqslant q_{i}\left\{\begin{array}{l}n_{k}=\left\lfloor\frac{k}{\left|p_{i}\right|}\right\rfloor, \\ n_{l}=l-\frac{\left(k-n_{k}\right)}{p_{i}} q_{i}\end{array}\right.$ otherwise $\left\{\begin{array}{l}n_{l}=\left\lfloor\frac{l}{\left|q_{i}\right|}\right\rfloor \\ n_{k}=k-\frac{\left(l-n_{l}\right)}{q_{i}} p_{i}\end{array}\right.$

(where $\lfloor x\rfloor$ gives the greatest integer less than or equal to $x$ ), such that the corresponding bin in the $1 \mathrm{D}$ projection, $\operatorname{proj}_{p_{i}, q_{i}}(b)$, is found as

$b=n_{l} p_{i}-n_{k} q_{i}$

This implies that 2D image compression schemes can be applied when performing intra-projection coding. The prediction complexity can be increased from a simple DPCM of order 1 to DPCM order to 3 and further to well known ADPCM techniques with context coding such as LOCO (of order 3) [17] and CALIC (of order 7) [18] and Glicbawls [12] which uses the entire set of causal data. As an example, the compression results in bpp for each of

\section{a}

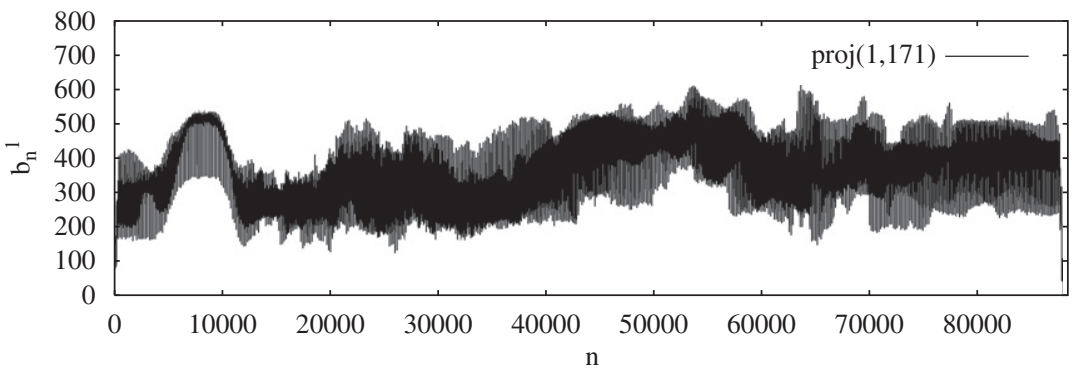

ii

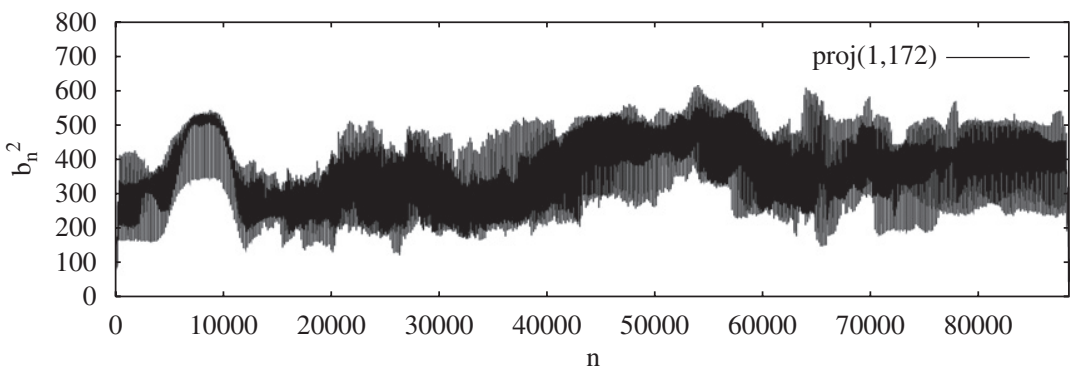

iii

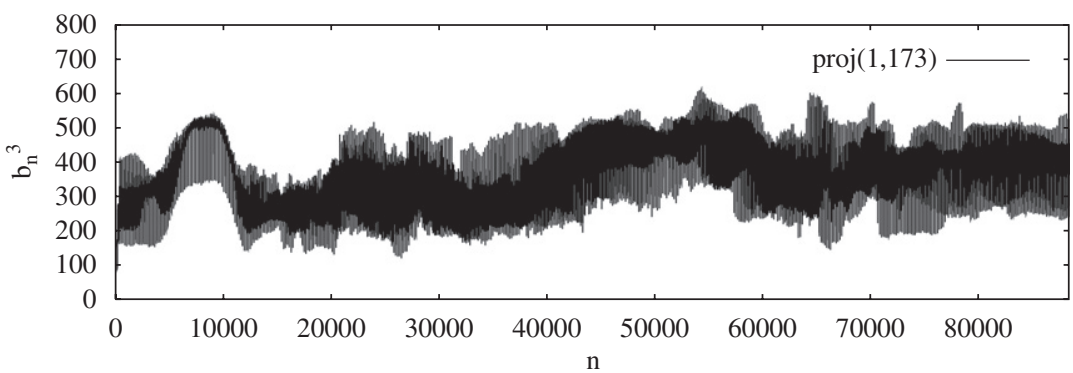

Fig. 5. (a) Three $1 \mathrm{D}$ projections of 'Lena' with projection vectors $\left(1, q_{i}\right)$ where $q_{i}$ is (i) 171 , (ii) 172 , and (iii) 173 . (b) The same projection data displayed as images with columns heights of $q_{i}$ and a width of 514 . 
b

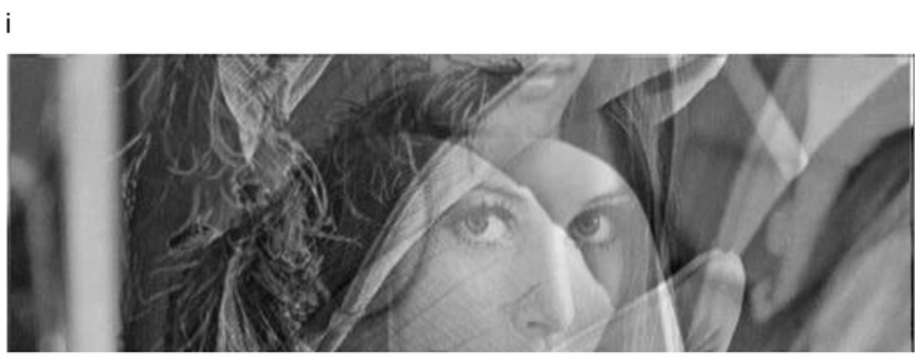

ii

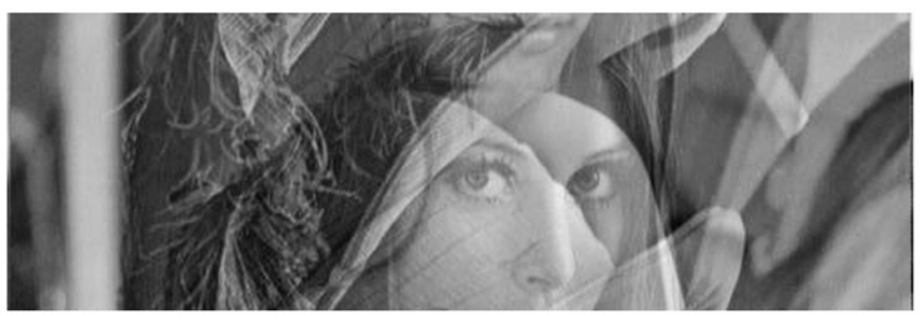

iii

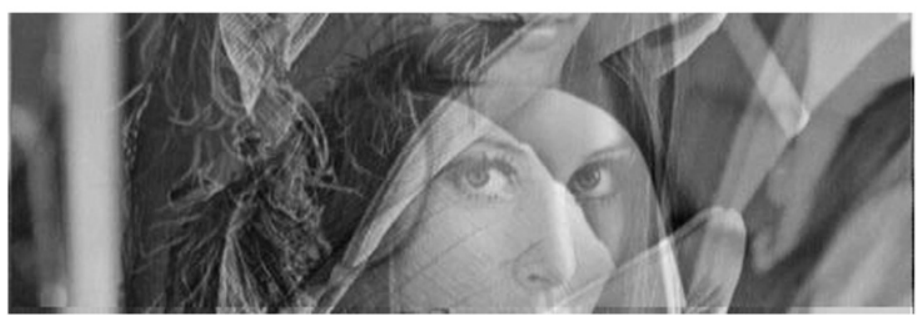

Fig. 5. (Continued)

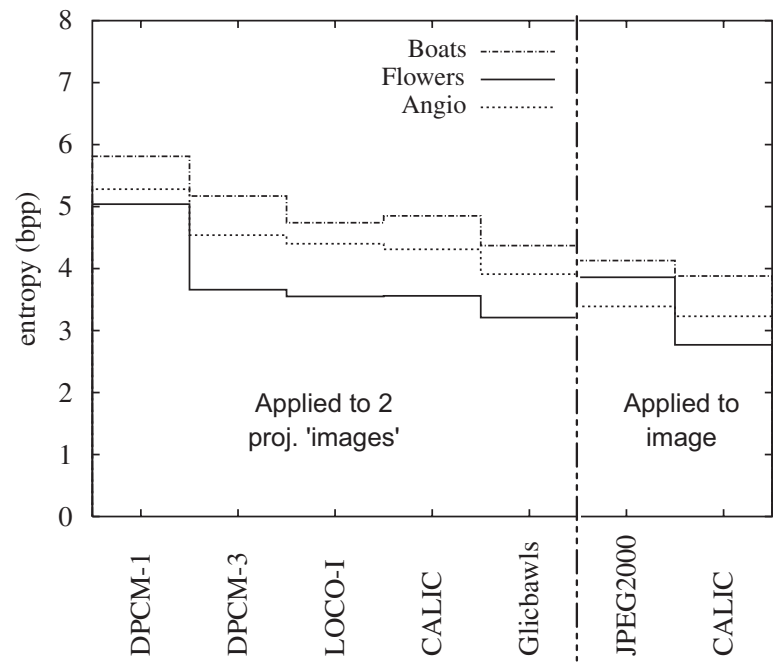

Fig. 6. The entropy results for intra-projection compression of two projection 'images' using prediction schemes: DPCM-1, DPCM-3, LOCO, CALIC, and Glicbawls. This is compared with applying JPEG2000 and CALIC directly to the image.

these respective methods to encode two projections of three different images using the direction vectors $\{(P / 2,1),(P / 2+1,1)\}$ is given in Fig. 6 and compared with the result of applying JPEG2000 to the image. (Similar results are achieved using the direction vectors $\{(1, Q / 2),(1, Q / 2+1)\})$. This shows that compression results better than JPEG2000 can be achieved in some cases with the use of more sophisticated image coding techniques without the need for inter-projection coding. This is an important result for distributed storage. However, the more complex techniques require more memory and computation time. A good trade-off (by design) is CALIC.

The implications of these results are that redundancy can be inserted for distributed storage or transmission very efficiently. For the example above, an extra projection with a direction vector $(-P / 2-1,1)$ can be also included. The resulting compression will still be less than $6.0 \mathrm{bpp}$ (as shown in Fig. 7 for 'Flowers') but with the added advantage that any two of the three projections is sufficient to recover the image according to (4). If each projection is stored on a unique server or transmitted over a unique channel, this is a very secure distribution scheme. Of course the number of projections required for reconstruction and the degree of redundancy can be tuned as required.

Fig. 7 gives the compression achieved with the number of projections required to recover the 'Flowers' image, $N$. At each $N$ there are three values, the first is the compression attained without redundancy in the 


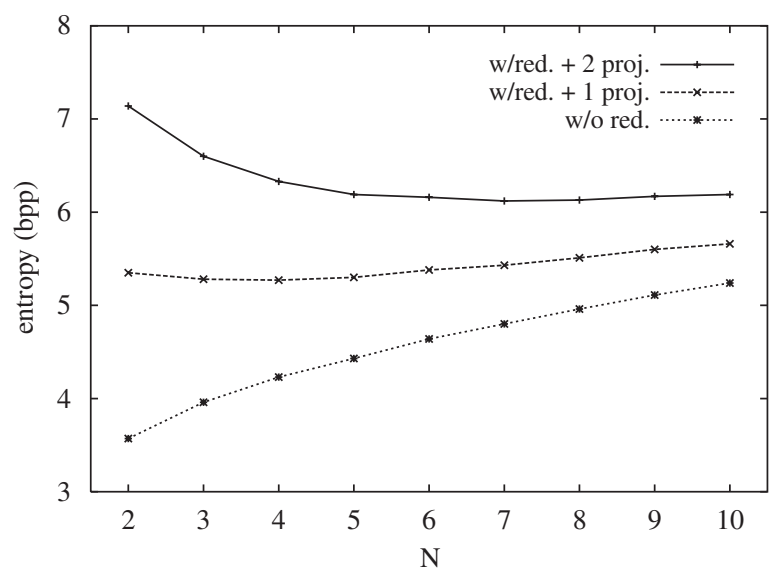

Fig. 7. A plot of compression against the number of projections, $N$, required to reconstruct the 'Flowers' image: without redundancy, with one extra projection (so any $N$ of the $N+1$ projections is sufficient for reconstruction), and with two extra projections (so any $N$ of the $N+2$ projections is sufficient for reconstruction).

\section{Current band, $\mathrm{i} \quad$ Reference band, i+1}
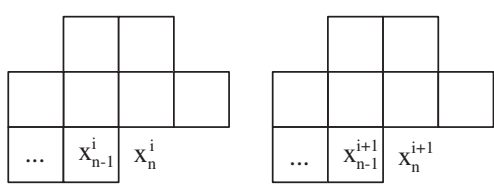

Fig. 8. The causal 'neighbourhood' in both the current spectral band and the reference spectral band used in inter-band CALIC prediction.

projections, i.e., $N$ projections. The next two give the compression attained with some redundancy, where 1 (resp. 2) extra projection(s) are included such that any $N$ of the $N+1$ (resp. $N+2$ ) projections are sufficient to recover the image, i.e., an additional redundancy of $1 / N$ (resp. $2 / N$ ).

Since the projections can be represented as images, inter-projection coding could be considered to be similar to inter-band image coding, e.g., coding between the RGB components of an image. The next section investigates inter-projection compression issues and techniques associated with this idea.

\subsection{Inter-projection compression}

A simple but effective inter-band prediction method is presented in [19] to extend CALIC to multi-spectral images. The essential idea is to compute the crosscorrelation between the current band, $x^{i}$, and the next band, $x^{i+1}$, over the causal 'neighbourhood' data used in CALIC prediction. These regions are depicted in Fig. 8; note that $x_{n}^{i}$ and $x_{n}^{i+1}$ have the same spatial location but are in different bands. If these two regions are highly correlated then the intra-band CALIC prediction of the $n$th pixel value of the current band, $\widehat{x}_{n}^{i}$, can be improved upon by using information from the next band, $x^{i+1}$. This is true since the actual value of the $n$th pixel in the reference band, $x_{n}^{i+1}$, is known. a

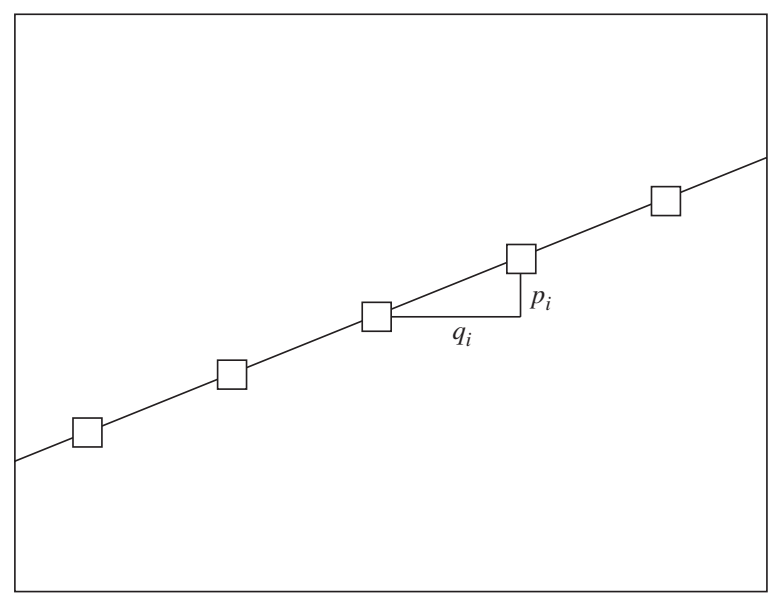

b

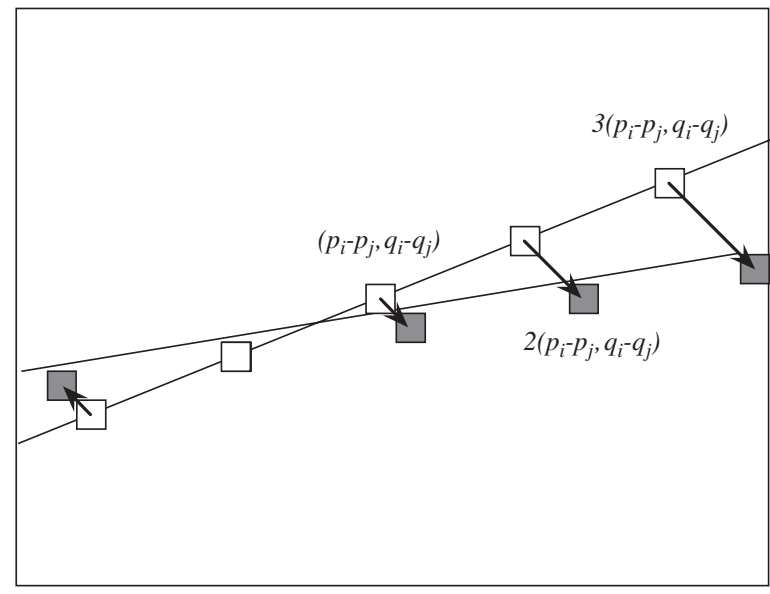

C

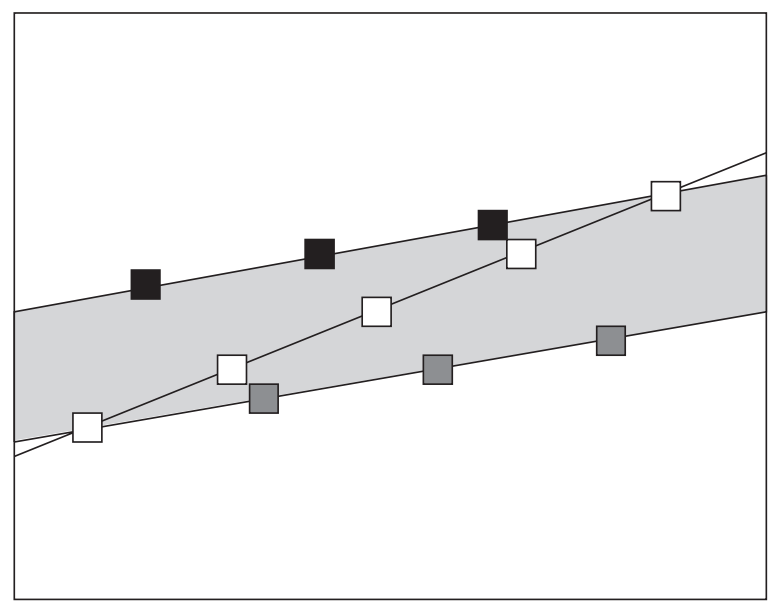

Fig. 9. (a) Relative position of pixel values summed to give a projection bin value. (b) The difference in sampled pixels between two raysums of different projections including a common pixel. (c) The shaded region contains raysums from $\operatorname{proj}_{p_{j}, q_{j}}$ that intersect the given raysum from $\operatorname{proj}_{p_{i}, q_{i}}$ whose contributing pixels are shown as white squares. 
A similar idea can be applied between Mojette projections of an image. In inter-band CALIC the different bands contain images that are spatially consistent but with different intensities according to the band of the spectrum. With inter-projection compression, however, the same pixel intensities, i.e., $f(k, l)$, exist in all projections but with a different phase or period of 'mixing' with other intensities. Therefore, the best phase to use when predicting each bin value must be determined. In other words, which projection, $\operatorname{proj}_{p_{j}, q_{j}}$, should be used as the reference projection and which bin in this projection best corresponds to the current bin required to be predicted in the current projection, $\operatorname{proj}_{p_{i}, q_{i}}$ ?

Let us investigate the position of pixels summed in a 'raysum' to give a bin value, $\operatorname{proj}_{p_{i}, q_{i}}(b)$. Adjacent pixels in this set are separated by $\left(p_{i}, q_{i}\right)$ as shown in Fig. 9a. Consider the pixels sampled by two raysums from different projections as depicted in Fig. 9b. This figure shows that the distance between the previous and subsequent pixels sampled increases by $\left(p_{i}-p_{j}, q_{i}-q_{j}\right)$ each step. Therefore when coding $\operatorname{proj}_{p_{i}, q_{i}}$ the obvious choice of projection to utilise as a reference for interprojection compression is $\operatorname{proj}_{p_{j}, q_{j}}$ such that the length of this difference vector is minimised. This is denoted as $\operatorname{proj}_{p_{i+1}, q_{i+1}}$

The question of which raysum from this projection should be used for prediction of $b_{n}^{i}=\operatorname{proj}_{p_{i}, q_{i}}(n)$ is not so obvious since it is more content dependant. A selection criterion is required to determine the best raysum from $\operatorname{proj}_{p_{i+1}, q_{i+1}}$ in the shaded region of Fig. 9c that will give the best prediction for the raysum, $b_{n}^{i}$, shown. There are two questions: 1. How many candidate raysums are there in $\operatorname{proj}_{p_{i+1}, q_{i+1}}$ ? and 2 . What are their bin indices?

To address the first question, assume there are a maximum of $r_{i}$ image pixels sampled in the raysum to give $b_{n}^{i}$. The value of $r_{i}$ is found as $\min \left(\left\lceil P / p_{i}\right\rceil,\left\lceil Q / q_{i}\right\rceil\right)$ where $\lceil x\rceil$ gives the smallest integer greater than or equal to $x$. The longest possible vector between corresponding sampled pixels of the two lines is therefore $\left(r_{i}-1\right)$ $\left(p_{i}-p_{i+1}, q_{i}-q_{i+1}\right)$. This also gives the maximum possible vector between pixels on the two bounding lines in Fig. 9c. Thus, from (2) there are $M=(r-1)\left[\left(q_{i}-q_{i+1}\right) p_{i+1}-\left(p_{i}-\right.\right.$ $\left.\left.p_{i+1}\right) q_{i+1}\right]+1$ candidate raysums in the shaded region of Fig. 9c. For this example assume $M=5$ as shown in Fig. 10a.

To determine the indices of these candidate bins are straightforward. The raysum that samples pixels closest to the $l$ axis (dark grey pixels in Fig. 10a) intersects with the raysum giving $b_{n}^{i}$ (white pixels in Fig. 10a) in first region of the image, $m=0$, as indicated in Fig. 11a. Therefore, the $\left(n_{k}, n_{l}\right)$ position of these bins is identical in the two projection 'images' (as depicted in Fig. 10b), i.e.,

$\widetilde{b}_{n}^{i+1}=\operatorname{proj}_{p_{i+1}, q_{i+1}}\left(n_{l} p_{i+1}-n_{k} q_{i+1}\right)$.

This is for a set of $\left(p_{i}, q_{i}\right)$ direction vectors where $\left|p_{i}\right| \geqslant q_{i}$. The same equation applies to the case where $\left|p_{i}\right|<q_{i}$, however, here it is the raysum the samples pixels closest to the $k$ axis (black pixels in Fig. 10a).

In summary, the bin values from $\operatorname{proj}_{p_{i+1}, q_{i+1}}$ that are candidates to be used in the prediction of $b_{n}^{i}$ include $\widetilde{b}_{n}^{i+1}$

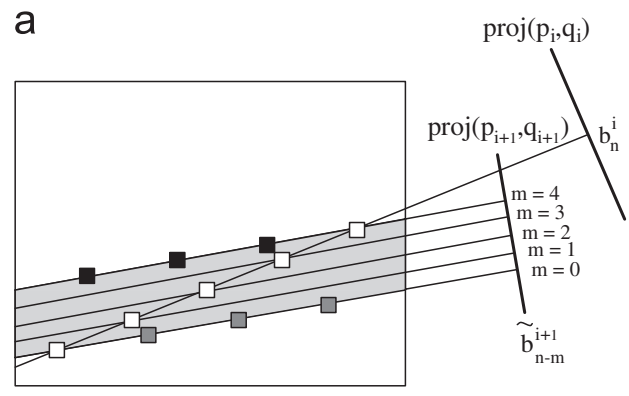

b

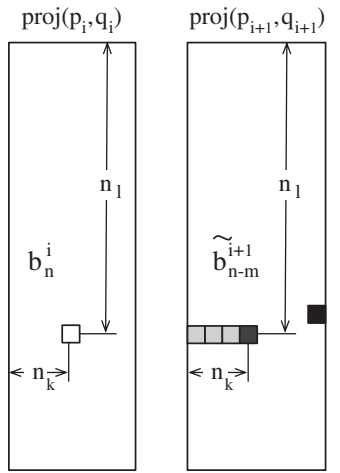

Fig. 10. (a) Depiction of the five candidate raysums from $\operatorname{proj}_{p_{i+1}, q_{i+1}}$ in the shaded region labelled by phase, $m$. Any of these could be used when predicting the given bin value in $\operatorname{proj}_{p_{i}, q_{i}}$. (b) The position of these bins in the respective projection 'images'.

from (10) and the preceding $M-1$ bin values, i.e., $\widetilde{b}_{n-m}^{i+1}$ for $m \in[0, M-1]$. These bins have been depicted in Fig. 10b for the example. Given the optimal candidate bin 'phase', $m$, the intra-projection prediction can be improved by

$\widehat{b}_{n}^{i}=\operatorname{pred}\left(b_{n}^{i}\right)+\widetilde{b}_{n-m}^{i+1}-\operatorname{pred}\left(\widetilde{b}_{n-m}^{i+1}\right)$,

where pred() is any of the prediction models introduced in Section 4.1.

Selecting phase, $m$, in the reference projection for the prediction, i.e., using $\widetilde{b}_{n-m}^{i+1}$, gives the best prediction for the $m$ th section of the image, as labelled in Fig. 11a for the example given in Fig. 10a and b. The prediction is successively worse for the regions $m \pm 1, m \pm 2$, and so on. A sensible choice for $m$ is therefore the raysum in the centre of the shaded region, $M / 2$, and this does in general give the best result. However, if the central region of the image is smoother than towards the image boundaries, the correct prediction of texture edge positions is more critical than an overall minimum distance between corresponding sampled pixels of the two raysums.

This has been demonstrated for several $512 \times 512$ images using the $\operatorname{proj}_{104,1}$ to predict projection $\operatorname{proj}_{103,1}$. In this case (as in the previous diagrams), there are $M=5$ candidate raysums to investigate which correspond to the five sections of the image as shown in Fig. 11a. In these regions the sampled pixels of the raysums from $b_{n}^{i}$ and $\widetilde{b}_{n-m}^{i+1}$ are identical (and hence the data of these sections is removed entirely according to (11)). Fig. 11b gives the inter-projection compression rates achieved using (11) 
a

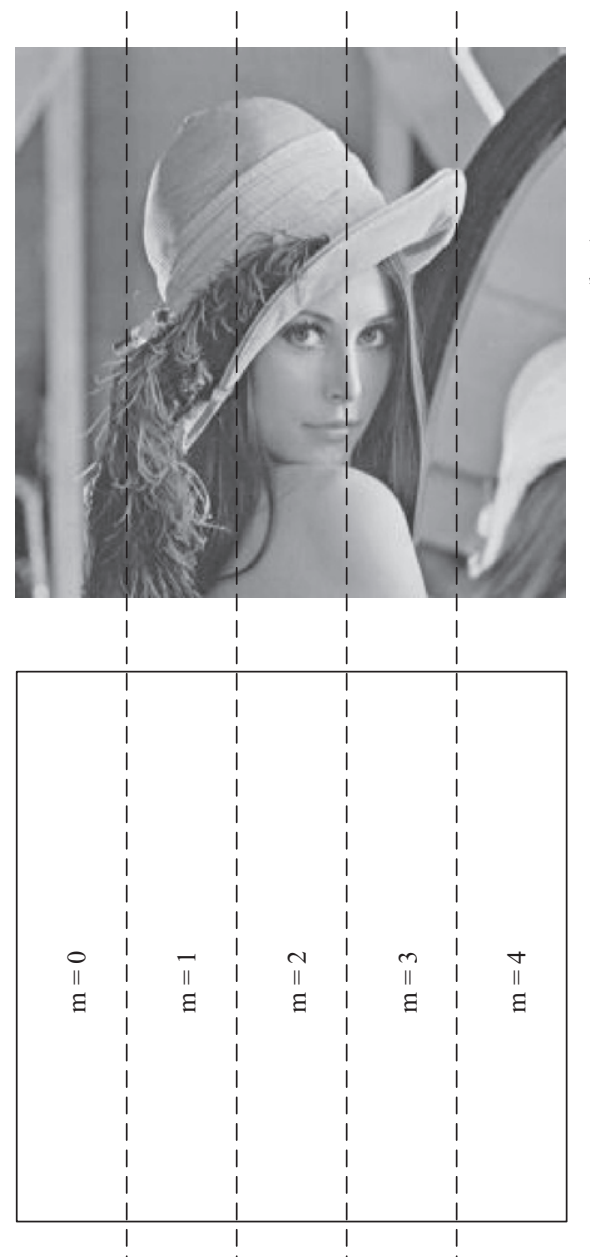

\section{b}

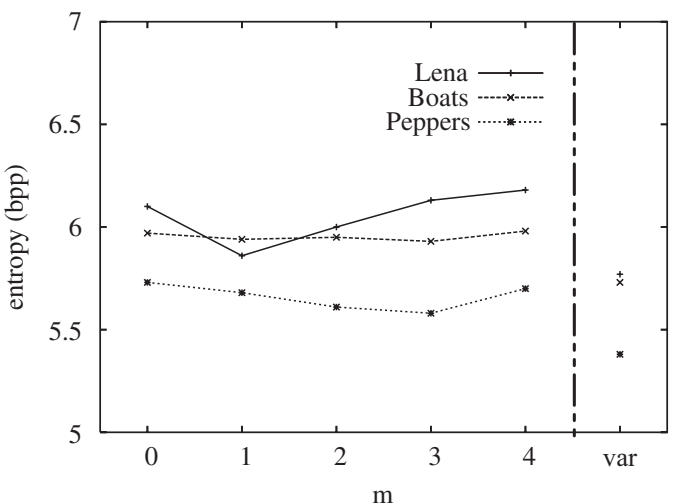

C

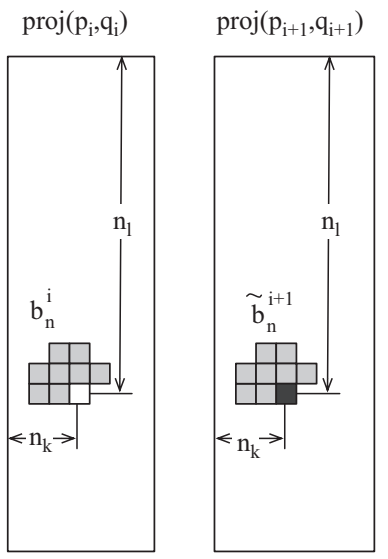

Fig. 11. (a) The five regions of the image which in selecting phase, $m$, in prediction will be removed entirely from the current projection after coding. (b) The compression results for inter-projection compression of $\operatorname{proj}_{103,1}$ from $\operatorname{proj}_{104,1}$ using constant $m$ and variable $m$, i.e., 'var'. (c) The causal neighbours from the current projection and the reference projection used firstly to determine cross-correlation and also for prediction.

with constant phase, $m$, for all predictions. Here pred() is the gradient adjusted prediction (GAP) introduced in [18]. It is expected that these plots attain a minimum at $m=2$, however, this is not true in practise for any of the plots.

By selecting phase, $m$, the contribution of the $m$ th section of the data is removed. Thus, better compression results are achieved when the phase corresponds to the most textured region of the image. For example, the second region of 'Lena', labelled $m=1$ in Fig. 11a, contains the highly textured hair. Removing this textured region from the projection by setting $m=1$ for inter-projection coding gives the lowest entropy as seen in Fig. 11b. These results demonstrate that the accurate location of texture edges is very important in the predictors performance.

A content dependant method is desired to select the optimum raysum from the $M$ candidate raysums of $\operatorname{proj}_{p_{i+1}, q_{i+1}}$. A fast method that attains near optimal performance in inter-projection coding is to determine the vertical region of the image (or horizontal for a set of $\left(1, q_{i}\right)$ direction vectors) with maximum 'activity' and select the phase of $\widetilde{b}_{n-m}^{i+1}$, accordingly.
A slower but more effective selection criteria can be achieved by investigating the 2D cross-correlation between the causal neighbourhoods of $b_{n}^{i}$ and $\widetilde{b}_{n-m}^{i+1}$ in the two projections for all phases, $m \in[0, M-1]$. These causal neighbourhoods have been depicted in Fig. 11c. The phase, $m$, of the candidate bin selected, $\widetilde{b}_{n-m}^{i+1}$, is that with the greatest cross-correlation with $b_{n}^{i}$. If this cross-correlation is above some threshold it should be useful to improve the intra-projection prediction, otherwise only intra-projection coding is considered. This gives the best overall compression results as shown for the 'var', (i.e., variable), column in Fig. 11b with compression consistently lower than any constant phase, $m$.

Fig. 12b shows, for the example projections, that interprojection coded data (12b(i) and (ii)) has been effectively decorrelated from the basis projection (12b(iii)). Fig. 12a shows the compression rates using GAP for inter-projection prediction and CALIC for intra-projection coding are comparable to JPEG2000. Fig. 13a and b summarises the results achieved in this paper for intra- and interprojection coding, respectively. They plot the average 
a
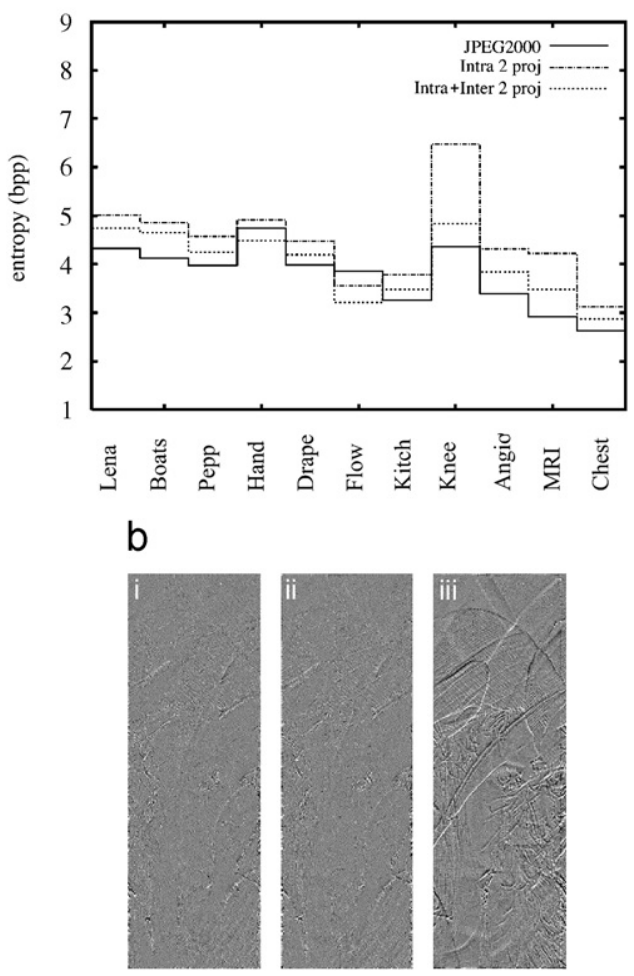

Fig. 12. (a) The compression results for inter-(GAP) and intra-(CALIC) projection compression of two projections using variable phase, $m$. Compared with the inter-projection compression results from the previous section using scheme $B$. (b) A visualisation of the decorrelation achieved using inter-projection coding. Three prediction error projections (i) $\operatorname{proj}_{170,1}$, (ii) $\operatorname{proj}_{171,1}$, and (iii) $\operatorname{proj}_{172,1}$ of 'Lena'. Projections (i) and (ii) have been inter-coded and effectively decorrelated from projection (iii). Note that the grey levels are centered about 0 with a window of 64 . compression ratios of each type of image, i.e., natural, art, and medical, using the compression techniques from the preliminary study (Fig. 4a), and the multi-spectral band image coding scheme (Fig. 12a). The average entropy of each scheme is compared with the entropy of the original image and the compression achieved using JPEG2000. Results show that this scheme is particularly suited to the intended application to the scanned art image database of the C2RMF. For the art images, the compression achieved using solely intra-projection coding is similar to JPEG2000 and including inter-projection coding is more effective than JPEG2000.

\section{Conclusions and future research}

The technique to losslessly compress images via linear prediction of the Mojette projection presented in [1] has been improved upon here. Average compression achieved using intra-projection coding of two projections was improved from 5.38 to 4.48 bpp using a fast lossless image coding technique. Average inter-projection compression entropy was also improved from 4.77 to $4.00 \mathrm{bpp}$ using a lossless inter-spectral band image compression technique. Fig. 13 shows that these improved results are comparable to those achieved using JPEG2000 applied directly to the image and that these techniques are particularly suited to the intended purpose of this work on compressing scanned art images for the TSAR project.

The image coding techniques have been adapted to fully exploit the nature of the Mojette projection data. This periodic nature is present since the Mojette projections preserve the $2 \mathrm{D}$ auto-correlation of the image and implies that image compression and inter-spectral band image compression can be applied. The prediction method selected for intra-projection coding should be selected

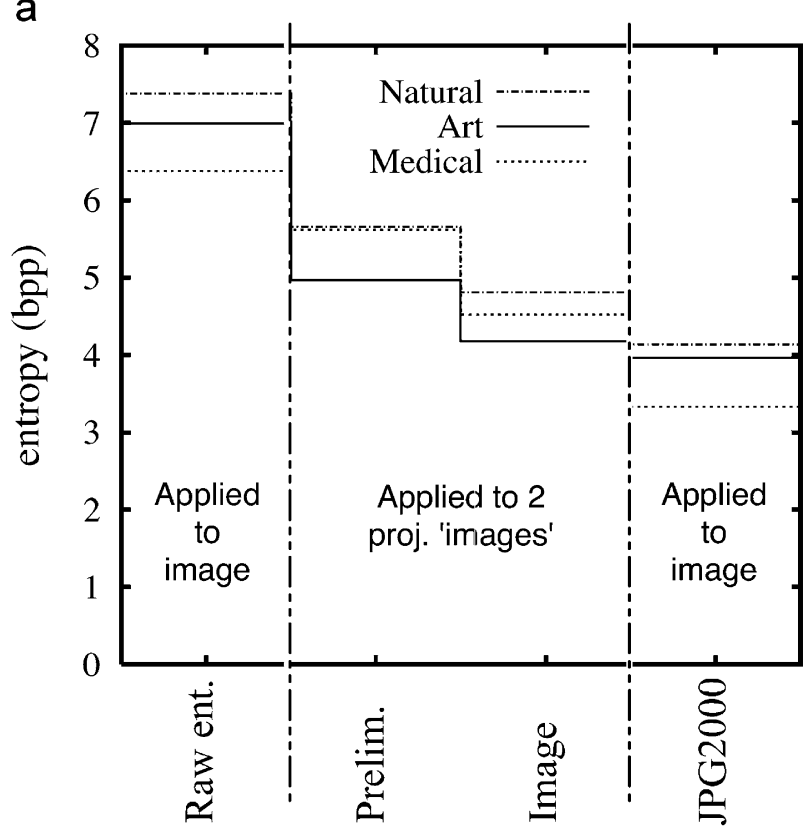

b

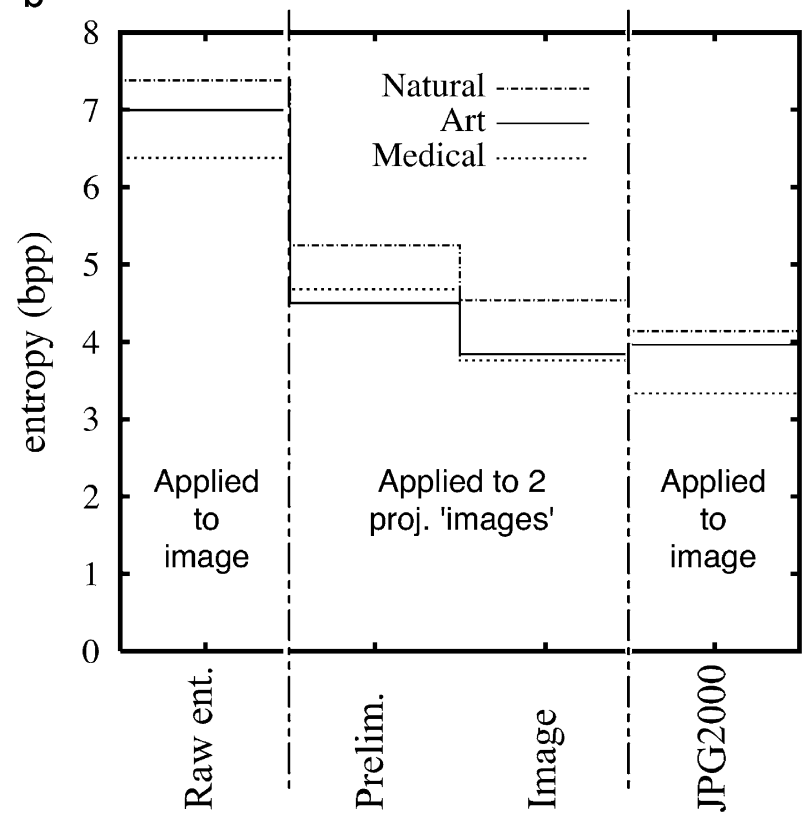

Fig. 13. A plot of average compression ratio over the 11 test images for both (a) intra- and (b) inter-projection coding using: the DPCM- 1 method from the preliminary study, and the CALIC image scheme. Results are compared with the average entropy of the raw image and the compression achieved by applying JPEG2000 to the image. 
depending on the requirements for compression and implementation time. A method to select the optimal raysum from all candidate raysums of a reference projection to use for inter-projection prediction has also been presented.

Compression rates comparable to JPEG2000 are achieved using image coding techniques, however, the image coding techniques take advantage of the 2D correlation and hence, by design, have less complexity, require less memory, and thus have a lower implementation time. Another possible compression scheme that may be applicable is video inter-frame coding with motion estimation and is a direction for future research. Another area for future investigation is that of coding colour images with predictions using inter-projection and interband correlation simultaneously.

These results imply that the Mojette projections which have applications in distributed storage and encryption of images in databases can also be effectively losslessly compressed and many of the requirements of an image database outlined in Section 1 can be achieved using solely the Mojette transform.

The methods explored here concentrated on predictive coding. Techniques using scalable transforms such as DWT, DCT and FFT may prove useful. If a block based approach could be made feasible, then it may also be beneficial to investigate projections directed along and orthogonal to texture orientation.

\section{Acknowledgements}

The majority of this work was conducted while A.K. held a postdoctoral position at l'Université de Nantes supported by a grant from the Région Pays de la Loire, France. This work is partially supported by projects Miles, Région Pays de la Loire, and ANR-TSAR.

\section{References}

[1] F. Autrusseau, B. Parrein, M. Servières, Lossless compression based on a discrete and exact radon transform: a preliminary study, in: Proceedings of the IEEE International Conference on Acoustics, Speech, and Signal Processing (ICASSP), vol. II, May 2006, Toulouse, France, pp. 425-428.

[2] H. Bin, G.F. Elmasry, C.N. Manikopoulos, Joint lossless-source and channel coding using ARQ/go-back-(N, M) for image transmission, IEEE Trans. Image Process. 12 (12) (2003) 1610-1617.

[3] G. Davis, J. Danskin, Joint source and channel coding for image transmission over lossy packet networks, in: SPIE Conference on
Wavelet Applications of Digital Image Processing XIX, August 1996 pp. 376-387.

[4] S.R. Deans, The Radon Transform and Some of its Applications, revised ed., Krieger, Malabar, FL, 1993.

[5] J.P. Guédon, D. Barba, N. Burger, Psychovisual image coding via an exact discrete Radon transform, in: T.W. Lance (Ed.), Proceedings of the Visual Communications AND Image Processing (VCIP), May 1995, Taipei, Taiwan, pp. 562-572.

[6] J.P. Guédon, N. Normand, The Mojette transform: the first ten years, in: G.É. Andres, G. Damiand, P. Lienhardt (Eds.), Proceedings of the 12th International Conference on Discrete Geometry for Computer Imagery, Lecture Notes in Computer Science, vol. 3429, Springer, Poitiers, France, 2005, pp. 79-91.

[7] P. Guédon, B. Parrein, N. Normand, Internet distributed image information system, Integrated Comput. Aided Eng. 8 (2001) 205-214.

[8] M. Katz, Questions of Uniqueness and Resolution in Reconstruction from Projections, Lecture Notes in Biomathematics, Springer, Berlin, 1977.

[9] B.T. Kelley, V.K. Madisetti, The fast discrete Radon transform. I: theory, IEEE Trans. Image Process. 2 (3) (1993) 382400.

[10] A. Kingston, S. Colosimo, P. Campisi, F. Autrusseau, Lossless image compression and selective encryption using a discrete radon transform, in: IEEE International Conference on Image Processing September 16-19 2007, San Antonio, TX, USA

[11] F. Matúš, J. Flusser, Image representation via a finite Radon transform, IEEE Trans. Pattern Anal. Mach. Intell. 15 (10) (1993) 996-1006.

[12] B. Meyer, P. Tischer, Glicbawls - grey level image compression by adaptive weighted least squares, in: IEEE Computer Society, Proceedings of the Data Compression Conference, Snowbird, Utah, USA, March 2001, p. 503.

[13] Y. Ming, N. Bourbakis, An overview of lossless digital image compression techniques, in: 48th Midwest Symposium on Circuits and Systems, vol. 2, 7-10, August 2005, pp. 1099-1102.

[14] N. Normand, J.P. Guédon, O. Philippé, D. Barba, Controlled redundancy for image coding and high-speed transmission, in: R. Ansari, M.J. Smith (Eds.), Proceedings of the SPIE Visual Communications and Image Processing, vol. 2727, February 1996 , pp. 1070-1081.

[15] N. Normand, A. Kingston, P. Évenou, A geometry driven reconstruc tion algorithm for the Mojette transform, in: A. Kuba, L.G. Nyúl, K. Palágyi (Eds.), Proceedings of the 13th International Conference on Discrete Geometry for Computer Imagery, Lecture Notes in Computer Science, vol. 4245, Springer, Szeged, Hungary, 2006, pp. 122-133.

[16] O. Philippé, J.P. Guédon, Correlation of the Mojette representation for non-exact image reconstruction, in: Proceedings of Picture Coding Symposium, vol. 1, Berlin, Germany, September 1997 pp. 237-241.

[17] M.J. Weinberger, G. Seroussi, G. Sapiro, LOCO-I: a low complexity, context-based, lossless image compression algorithm, in: Proceedings of the Data Compression Conference, IEEE Computer Society, Los Alamitos, CA, USA, March-April 1996, pp 140-149.

[18] X. Wu, N. Memon, Context-based, adaptive, lossless image coding, IEEE Trans. Comm. 45 (4) (1997) 437-444.

[19] X. Wu, N. Memon, Context-based lossless interband compressionextending CALIC, IEEE Trans. Image Process. 9 (6) (2000) 994-1001.

[20] F. Zhai, Y. Eisenberg, A.K. Katsaggelos, Joint source-channel coding for video communications, in: Al. Bovik (Ed.), Handbook of Image and Video Processing, second ed., Elsevier Academics Press, USA 2005 